\title{
Erratum zu: Was lernt man in Trainingsgruppen? Auswirkungen erfahrungsorientierter Lernsettings auf die Entwicklung von Schlüsselkompetenzen
}

\author{
Jonas Claußen
}

Online publiziert: 11. Januar 2016

(C) Springer Fachmedien Wiesbaden 2015

\section{Erratum zu:}

Gruppendyn Organisationsberat (2015)

DOI 10.1007/s11612-015-0295-1

Im Kap. 5.1.5 Statistische Auswertungen wurde angegeben, dass eine zweifaktorielle Varianzanalyse mit Messwiederholung auf beiden Faktoren durchgeführt wurde. Die

In Kap. 5.2 dargestellten Ergebnisse beziehen sich jedoch auf die durchgeführten Berechnungen mittels t-Tests bei abhängigen Stichproben. Demnach muss der zweite Satz in Kap. 5.1.5 korrektermaßen lauten: Bei der SPSS Analyse der Testergebnisse wurden für den vorliegenden Beitrag im ersten Schritt t-Tests mit abhängigen Stichproben durchgeführt.

Die Online-Version des Originalartikels ist unter doi:10.1007/s11612-015-0295-1 zu finden

MMag. J. Claußen $(\bowtie)$

Institut für Organisationsentwicklung, Gruppendynamik und Interventionsforschung, Alpen-Adria-Universität Klagenfurt, Sterneckstraße 15,

9020 Klagenfurt, Österreich

E-Mail: jonas.claussen@aau.at 